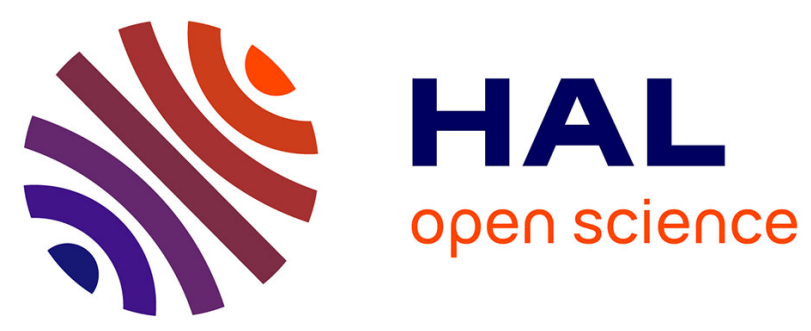

\title{
Optimization and control of bio-conversion of polymeric substrate in the chemostat
}

\author{
Jacques-Alexandre Sepulchre, Francis Mairet, Jean-Luc Gouzé
}

\section{To cite this version:}

Jacques-Alexandre Sepulchre, Francis Mairet, Jean-Luc Gouzé. Optimization and control of bioconversion of polymeric substrate in the chemostat. AIChE Journal, 2017, 63 (11), pp.4738-4747. 10.1002/aic.15853 . hal-01569696

\section{HAL Id: hal-01569696 \\ https://hal.inria.fr/hal-01569696}

Submitted on 27 Jul 2017

HAL is a multi-disciplinary open access archive for the deposit and dissemination of scientific research documents, whether they are published or not. The documents may come from teaching and research institutions in France or abroad, or from public or private research centers.
L'archive ouverte pluridisciplinaire HAL, est destinée au dépôt et à la diffusion de documents scientifiques de niveau recherche, publiés ou non, émanant des établissements d'enseignement et de recherche français ou étrangers, des laboratoires publics ou privés. 


\title{
Optimization and control of bio-conversion of
}

\section{polymeric substrate in the chemostat}

\author{
J-A. Sepulchre ${ }^{1}$ and F. Mairet ${ }^{2}$ and J-L. Gouzé ${ }^{2 *}$
}

May 30, 2017

\begin{abstract}
We design a simple model of the growth of polymer-decomposing bacteria in a continuous stirred tank reactor. The dilution rate is controled to optimize the output of monomers. This model is studied in order to find the optimum at the stationary state. However, the optimal stationary state is not robust against perturbations, leading to washout in the bioreactor. We design a control that makes the closed system globally stable around the optimal equilibrium. Then, a more complex model for polymers and oligomers of any lengths is studied. It is shown that the same technics also lead to a globally stable optimal point for the controlled system.
\end{abstract}

Keywords: Nonlinear control, bioreactor, yield optimization, pathogenic bacterium.

\footnotetext{
*1 Université Côte d'Azur, Institut de Physique de Nice, CNRS - UMR 7010,

1361 route des lucioles, 06560 Sophia Antipolis, France, ${ }^{2}$ Université Côte d'Azur, IN-

RIA, BIOCORE project-team, 2004 Route des Lucioles, BP 93, 06902 Sophia Antipolis, France, jacques-alexandre.sepulchreeinphyni.cnrs.fr, francis.maireteinria.fr, jean-luc.gouzedinria.fr
} 


\section{Introduction}

One of the most abundant macrobiomolecule at the surface of earth is the cellulose ${ }^{1}$. Cellulose is a polycarbohydrate forming an important constituent of the cell wall of green plants, and can thus be considered as the main part of the biomass. Cellulose belongs to the family of polysaccharides, i.e. polymers of sugars. Its monomer is the high-energy molecule of glucose which can be fermented into alcool-based fuel, e.g. ethanol. Therefore, the decomposition of the cellulose found in the vast biomass waste into glucose offers, in principle, a great potential to produce precursor molecules for biofuel. Indeed, the conversion of the cellulose into ethanol has been recognized as a potential source of fuel, more than a century ago ${ }^{2}$. Since then, this idea has attracted a lot of investments, first in the context of biofuels of first generation, and next, as a possible way to recycle biomass wastes, leading its industrial development to produce the so-called biofuels of second generation ${ }^{2-4}$.

Other polycarbohydrates than cellulose found in biomass wastes possess also a potential of reconversion into precursor molecules of biofuels. An example is given by the pectins which, after celluloses and hemi-celluloses, can be considered as the third main structural polysaccharide group of plant cell walls ${ }^{3}$. The primary component of pectins is polygalaturonic acids whose decomposition can give a sugar called the galacturonate ${ }^{5}$.

In practice, a problem met by the industry to profitably produce biofuel of second generation is to have at its disposal efficient enzymes, able to decompose the considered polysaccharides ${ }^{6,7}$. One way to tackle this issue is to use specific bacteria that are specialized in the degradations of polysaccharides, because they produce the appropriate degradative enzymes able to lyse the considered polymers. Then, one can envisage to design a continuous process taking place into a bioreactor where bacteria secreting 
the specific enzymes are cultivated, continuously decomposing the polymeric substrate entering in the chemostat into monomeric sugars that are extracted from the system for a subsequent fermentation.

In this context, examples of efficient micro-organisms with cellulolysic capabilities have been recently pointed out ${ }^{8}$. Regarding the decomposition of pectins, efficient bacteria are well studied since a long time in the context of phytopathogenic microbiology ${ }^{9,10}$. In both cases, these bacteria are specialized in decomposing a given source of carbohydrates by secreting degradative enzymes for respectively cellulolysis or pectinolysis. Now, whatever the polysaccharides and the corresponding degradative enzymes synthesized by bacteria, their economical benefits depend on the possibility to optimize, as far as possible, the parametric conditions of the bioreactor. The goal is to maximise the outflow of mono-saccharides obtained from the decomposed polysaccharides. In the case where the bacteria consume the mono-saccharides for their own growth, one can anticipate that the sought optimization depends on two competitive processes. One the one hand, one wishes to maximize the extraction of the monomer which is produced thanks to the bacteria. On the other hand, the monomer is at the same time the limiting substrate of these bacteria. Consequently, as it will be shown below, the optimal steady state is unstable. A way to globally stabilize it is presented below.

In this paper, we first present a minimal mathematical model for the bioconversion of the polysaccharides into their monomers when the reactants are placed in a bioreactor. The latter contains the degradative bacteria and is fed in by the substrate to be decomposed. A continuous extraction of the bioreactor enables to keep a constant volume and to extract a medium containing the (partly) decomposed substrate, that is the monomers. Our model is minimal because only 3 variables are considered, namely the 
concentrations of bacteria, polysaccharides, and monomers. Next, an extension of the minimal model takes into account the intermediate products of all the polymers and oligomers of intermediate sizes between the full-length polymer and the monomers. Both models are ruled by standard equations for describing the bacterial growth in presence of substrate. The minimal model, generalizing the Monod model ${ }^{11}$, was also considered by other authors ${ }^{12}$. However, we analyse it in a different perspective of optimization and control. The optimal equilibrium point of functioning is reached when the outflow product of degradation is maximal. This maximal regime is a bifurcation point and is not robust with respect to perturbations, therefore leading to washout of the bioreactor. Considering the dilution rate $D$ as the control, we are able to build a closed loop feedback depending on the measurements of bacterial concentration (through e.g. the optical density) that makes the closed system globally stable around the optimal equilibrium point. The same procedure applies when considering the more complex model with full-length polymers and intermediate oligomers.

From a Control point of view, the problem is rather difficult because the system is non-linear, and the existing results concerning global stabilizability are not so numerous; moreover, there are constraints of positivity on the control variable, that makes the problem still more difficult ${ }^{13,14}$. Our control law is inspired by our works for stabilization of bioreactors ${ }^{15,16}$. In the next section, the 3 -variable model is first presented and briefly studied. Then the optimal equilibrium is computed (Section 3) and the closed loop law built (Section 4). Finally, the detailed model with fragmentation of polymers and its stabilizing control is built (Section 5 and 6 ). Some simulations showing the efficiency of our approach are shown (Section 7). 


\section{The model}

First, the simplified model is presented with the aim of controlling the dilution rate to optimize the bioconversion of the polymeric substrate. The bacteria are supposed to be cultivated in a bioreactor (continuous stirred tank reactor), also called chemostat at lab scale. This will allow the existence of a positive equilibrium, and applications of techniques from Control Theory ${ }^{17}$. The variables, as explained above, are the molar concentrations of a $n$-polymer, (denoted $\left.s_{n}\right)$, cleaved into monomers (denoted $\left.s_{1}\right)$, and $\rho$ is the bacterial biomass.

The physical input is a flux of polymer (of molar concentration $s_{i n}$ ), and the controlled input (action on the system) will be the dilution rate $D$. The dynamical equations are:

$$
\begin{aligned}
\frac{d s_{n}}{d t} & =-\alpha\left(s_{n}\right) \rho+D\left(s_{i n}-s_{n}\right) \\
\frac{d s_{1}}{d t} & =n \alpha\left(s_{n}\right) \rho-\gamma \mu\left(s_{1}\right) \rho-D s_{1} \\
\frac{d \rho}{d t} & =\left(\mu\left(s_{1}\right)-D\right) \rho
\end{aligned}
$$

with

$$
\alpha\left(s_{n}\right)=\bar{\alpha} \frac{s_{n}}{K_{M}+s_{n}} \quad \text { and } \quad \mu\left(s_{1}\right)=\bar{\mu} \frac{s_{1}}{K_{1}+s_{1}} .
$$

The term $\alpha\left(s_{n}\right) \rho$ represents the one-step conversion of polymer $s_{n}$ into $n$ monomers $s_{1}$, catalyzed by the enzyme whose external concentration is taken proportional to biomass $\rho$. In particular, parameter $\bar{\alpha}$ determines the maximal rate of enzyme decomposition as being $\bar{\alpha} \rho$. The cells grow on the $s_{1}$ substrate (term $\mu\left(s_{1}\right) \rho$ ) with a maximal growth rate equal to $\bar{\mu}_{1}$. Parameter $\gamma$ is the yield between substrate $s_{1}$ and biomass production. 


\section{Steady states of the chemostat}

The steady states of the system are briefly investigated (for more details see ${ }^{12}$ ). There always exists a equilibrium with $s_{n}=s_{i n}, s_{1}=0$ and $\rho=0$ that corresponds to the chemostat washout with no bacteria. That trivial equilibrium is locally stable. Depending on the values of parameters, other positive equilibria may exist. Figure 1 illustrates a typical bifurcation diagram of the steady states in function of the dilution rate $D$. This diagram reveals the existence of a saddle-node bifurcation where two branches of stable and unstable equilibria merged for a limit value $D=D_{c}$. Let us remark that the steady state value of the monomer $s_{1}^{*}$ depends on $D$ in a simple way (deduced from $\dot{\rho}=0)$ :

$$
s_{1}^{*}(D)=\frac{D K_{1}}{\bar{\mu}-D}
$$

and therefore the stable and unstable branches of stationary values of $s_{1}^{*}$ are superposed into one. On the other hand, the two branches of equilibria of $s_{n}$ can computed by solving the following quadratic equation in $s_{n}^{*}$ :

$$
\left[1-\frac{\gamma D}{n \bar{\alpha}}\right] s_{n}^{* 2}+\left[\frac{\gamma D}{n \bar{\alpha}}\left(s_{i n}-K_{M}\right)-s_{i n}+\frac{D K_{1}}{n(\bar{\mu}-D)}\right] s_{n}^{*}+\frac{\gamma D}{n \bar{\alpha}} s_{i n} K_{M}=0
$$

Finally, the corresponding steady state values of $\rho^{*}$ can be deduced from the conservation law:

$$
n s_{n}^{*}+s_{1}^{*}+\gamma \rho^{*}=n s_{i n}
$$

This last equation is deduced straightforwardly from system (0.1) by making $n \dot{s}_{n}+$ $\dot{s}_{1}+\gamma \dot{\rho}=0$.

At the bifurcation value $D_{c}$ of the dilution rate, the discriminant of the quadratic eq. (0.3) cancels. The corresponding equation for $D$ is a polynomial of the 4 th degree, with generally no explicit solutions. However, in the case where $D \ll \bar{\mu}$, and considering the approximation $1 /(\bar{\mu}-D) \simeq 1 / \bar{\mu}$, one gets a simplified analytical 
expression of $D_{c}$ as follows :

$$
\mathcal{D}_{c}=\frac{n \bar{\alpha} s_{i n}}{\gamma} \frac{s_{i n}+\left(\sqrt{\frac{\bar{\alpha} K_{1}}{\gamma \bar{\mu}}}-\sqrt{K_{M}}\right)^{2}}{4 K_{M} s_{i n}+\left(s_{i n}+\frac{\bar{\alpha} K_{1}}{\gamma \bar{\mu}}-K_{M}\right)^{2}}
$$

Although in the next sections one will not reckon with the approximation $D \ll \bar{\mu}$, to derive our main results about optimal productivity and about its control, eq. (0.5) is useful to have an idea on how $D_{c}$ depends on the system parameters. Moreover an example of numerical application using eq. (0.5) with realistic parameters is illustrated on Fig. 1. The approximated limit points of the saddle-node curves are represented by symbols " $\mathrm{x}$ ". They reveal to be good estimates of the actual limit points obtained by numerical computations.

The model equations (0.1) were partly studied in ${ }^{12}$, with results on local and global stability. The key point is the remark that the system may be reduced in dimension two, because of the conservation law of the function $n s_{n}+s_{1}+\gamma \rho$.

\section{Optimal productivity}

The productivity of the system is the outgoing flux of monomers, $P=D s_{1}$, and the objective is to solve the problem of optimizing $P$ at the stationary state with $D$ as a control.

Theorem 1. The maximal steady-state productivity is obtained for $D=D_{c}$.

Proof. At steady-states, it can be deduced from $\dot{\rho}=0$ that the function $s_{1}^{*}(D)$ was given by eq. (0.2). Thus, for all $D$ such that there exists a non-trivial equilibrium (i.e. $\forall D \in\left(0 ; D_{c}\right]$, the productivity rewrites:

$$
P(D)=D s_{1}^{*}(D)=\frac{D^{2} K_{1}}{\bar{\mu}-D}
$$

$P(D)$ is an increasing function on $\left(0 ; D_{c}\right]$, so the result holds. 
Remark 1. Note that for a given dilution rate, the stable and the unstable equilibria have the same productivity.

Remark 2. The efficient functioning of the system can also be evaluated by dividing the productivity of the system by Dns ${ }_{i n}$, which is the maximum value it would have if all the polymeric substrate entering in the bioreactor was transformed into monomers. This quantity is called the efficiency $\eta$. This quantity can be computed as:

$$
\eta(D)=\frac{D K_{1}}{n s_{i n}(\bar{\mu}-D)}
$$

Obviously, the maximal steady-state efficiency is also obtained for $D=D_{c}$.

Our results show that the optimal control $D$ corresponds, on the bifurcation diagram of $s_{1}$ in function of $D$, precisely to the upper boundary point of the stable branch of equilibria; just after this point, the stable positive equilibrium disappears and only the washout point is stable (see Fig. (1)). Without control, any perturbation (for example a perturbation in the input $s_{i n}$ ) may lead to washout, that is of course not admissible from the industrial point of view. Therefore control is needed to make this positive equilibrium robustly stable against perturbations.

\section{Control for optimization}

As usual, the dilution rate $D$ is taken as the (positive) control variable. Assuming measurements of the biomass $\rho(t)$ (e.g. by optical density, which is also quite usual), let us consider the following control law:

$$
D(t)=\delta \rho(t),
$$

where $\delta$ is a positive fixed design parameter. 
Polymer

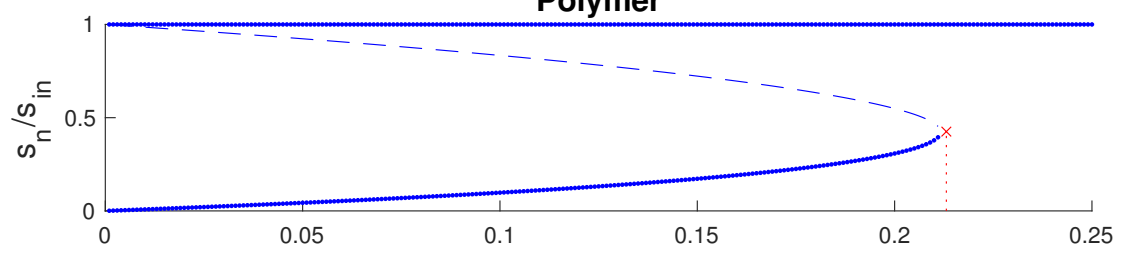

Monomer

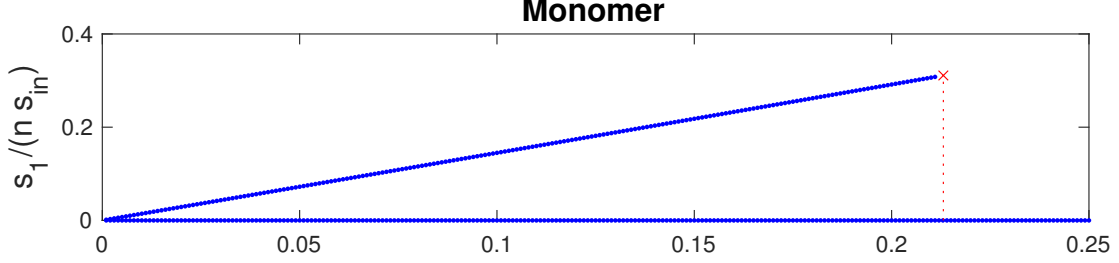

Biomass

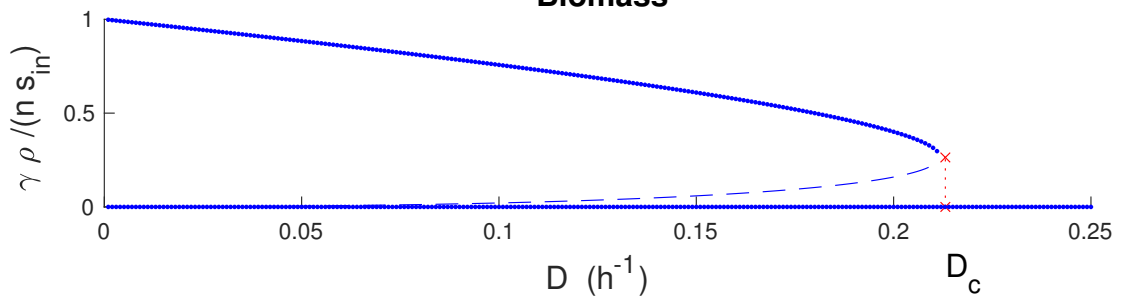

Figure 1: The bifurcation diagrams of the chemostat steady states show the existence of a limit point. The dashed curves are unstable equilibria, whereas the plain curves are stable equilibria. For the monomer variable, the non-zero stable and unstable equilibria are superposed on the same curve. The limit value $D_{c}$ is computed from the approximation $(0.5)$ and the corresponding limit points are drawn with symbols " $\mathrm{x}$ ". Parameters used in eqs (0.1) are partly taken from a study on the polygalacturonate decomposition by Dickeya dadantii ${ }^{10}$ and are the following : $\bar{\alpha}=12 \mathrm{~h}^{-1}, \bar{\mu}=17.1$ $\mathrm{h}^{-1}, \gamma=7.9 \mathrm{M}, s_{i n}=0.003 \mathrm{M}, K_{M}=6.810^{-3} \mathrm{M}, K_{1}=0.14 \mathrm{M}, n=2$. 
For $\delta>0$, let $s^{*}(\delta), s_{1}^{*}(\delta)$, and $\rho^{*}(\delta)$ the unique solutions on $\mathbb{R}_{*}^{+}$of respectively:

$$
\begin{aligned}
-\alpha\left(s_{n}^{*}\right)+\delta\left(s_{i n}-s_{n}^{*}\right) & =0, \\
2 \alpha\left(s_{n}^{*}(\delta)\right)-\gamma \mu\left(s_{1}^{*}\right)-\delta s_{1}^{*} & =0, \\
\mu\left(s_{1}^{*}(\delta)\right)-\delta \rho^{*} & =0 .
\end{aligned}
$$

Theorem 2. Control law (0.7) globally stabilizes (in the positive orthant) System (0.1) towards the non-trivial equilibrium $E^{*}(\delta):=\left(s_{n}^{*}(\delta), s_{1}^{*}(\delta), \rho^{*}(\delta)\right)$.

Proof. System (0.1) under Control law (0.7) becomes:

$$
\begin{aligned}
\frac{d s_{n}}{d t} & =\rho\left[-\alpha\left(s_{n}\right)+\delta\left(s_{i n}-s_{n}\right)\right] \\
\frac{d s_{1}}{d t} & =\rho\left[2 \alpha\left(s_{n}\right)-\gamma \mu\left(s_{1}\right)-\delta s_{1}\right] \\
\frac{d \rho}{d t} & =\rho\left[\left(\mu\left(s_{1}\right)-\delta \rho\right)\right]
\end{aligned}
$$

Because $\rho=0$ is invariant, $\rho$ never cancels in finite time for $\rho(0)>0$. Thus, from the dynamics of $s_{n}$, it is straightforward that $\forall t \geq 0$ :

$$
s_{n}(t) \geq \underline{s_{n}}:=\min \left(s_{n}^{*}(\delta), s_{n}(0)\right)
$$

and so $\frac{d s_{1}}{d t} \geq \rho\left[2 \alpha\left(\underline{s_{n}}\right)-\gamma \mu\left(s_{1}\right)-\delta s_{1}\right]$. It can be deduced that $s_{1}$ is lower bounded by $\underline{s_{1}}:=\min \left(s_{1}^{\dagger}, s_{1}(0)\right)$, where $s_{1}^{\dagger}$ is the unique solution on $\mathbb{R}_{*}^{+}$of $2 \alpha\left(\underline{s_{n}}\right)-\gamma \mu\left(s_{1}\right)-$ $\delta s_{1}=0$. It follows that:

$$
\frac{d \rho}{d t} \geq \rho\left[\left(\mu\left(\underline{s_{1}}\right)-\delta \rho\right)\right]
$$

and so $\rho(t) \geq \underline{\rho}:=\min \left(\frac{\mu\left(s_{1}\right)}{\delta}, \rho(0)\right), \forall t \geq 0$. This lower bound on $\rho(t)$ allows us to introduce the time change $\tau=\int_{0}^{t} \rho(x) d x$. System (0.8) rewrites:

$$
\begin{aligned}
\frac{d s_{n}}{d \tau} & =-\alpha\left(s_{n}\right)+\delta\left(s_{i n}-s_{n}\right) \\
\frac{d s_{1}}{d \tau} & =2 \alpha\left(s_{n}\right)-\gamma \mu\left(s_{1}\right)-\delta s_{1} \\
\frac{d \rho}{d \tau} & =\mu\left(s_{1}\right)-\delta \rho
\end{aligned}
$$


This system has a cascade structure, which means that the first equation is autonomous, and each following equation depends on its current variable which is $\tau$-differentiated, and on the variables appearing in the previous equations only. Such system is also said to be in triangular form and its global stability can be analysed as follows. The hypotheses of the theorem on stability of hierarchical systems ${ }^{18} \mathrm{ch} .5$, theorem 113 are verified because the system is autonomous and all the elements of the Jacobian matrix are bounded in the positive orthant, since the functions $\alpha$ and $\mu$ are of MichaelisMenten type. Next, one can easily show that $s_{n}$ asymptotically converges towards $s_{n}^{*}(\delta)$. Substituting $s_{n}$ by its equilibrium in the dynamics of $s_{1}$, it is deduced that $s_{1}$ asymptotically converges towards $s_{1}^{*}(\delta)$ in this limiting system. Similarly, using $s_{1}=s_{1}^{*}(\delta)$ in the dynamics of $\rho$, we obtain the global asymptotically convergence of $\rho$ towards $\rho^{*}(\delta)$, which concludes the proof.

Remark: Control law (0.7) is defined for all $\delta>0$. In the limits, we have:

$$
\lim _{\delta \rightarrow 0} E^{*}(\delta)=\left(0,0, \frac{n s_{i n}}{\gamma}\right) \text { and } \lim _{\delta \rightarrow+\infty} E^{*}(\delta)=\left(s_{i n}, 0,0\right)
$$

The limit $\delta \rightarrow 0$ is meant to be taken among the steady states of system (0.8). So the conservation law (0.4) enables to determine the limit of $\rho^{*}(0)=n s_{i n} / \gamma$ in this case. Now, referring the third panel of Fig.1, showing the $\rho^{*}(D) / \rho^{*}(0)$, one sees that each point of the steady state curve can be reached for one value of $\delta>0$. In particular $\delta$ can be adjusted so that the required equilibrium $E^{*}(\delta)$ corresponds to the optimal equilibrium. Thus the following theorem is also proved:

Theorem 3. In particular, it is possible to choose $\delta$ such that the closed loop system is globally stable around the optimal steady state.

Figure 2 shows an example of numerical results obtained for an interval of $\delta$ values. Each point of the curve of Fig. 2 is numerically computed by solving the differential 
equations (0.8) until it reaches its steady state and then plotting the resulting productivity $P$ as a function of $\delta$. In agreement with theorems 1 and 2, one sees that one can choose a value $\delta_{\text {opt }}$ which stabilises the chemostat on the steady state corresponding to the limit value $D_{c}$ yielding the optimal productivity.

The main advantage of our control method is to allow the chemostat working at its optimal point while avoiding the risk of washing out, which exists in the open system due to the bifurcation point. This property is illustrated in figure 3 , where one sees the effect of slightly fluctuating the input $s_{i n}$ of the chemostat. When the feedback loop is absent, and thus the dilution coefficient $D$ is constant, a temporary negative fluctuation of $s_{i n}$ can irremediably decrease the biomass under a threshold such that the bacteria decay even when the initial $s_{i n}$ is restored (dashed blue curve in fig. 3). On the contrary, applying the control method $D=\delta \rho$ enables that the biomass level is basically maintained during this negative fluctuation of the input and it recovers the optimal functioning when the initial input is re-established (continuous red curve in fig. 3).

\section{Fragmentation model}

In the system (0.1) studied in the previous section the enzymatic decomposition of a $n$-polymeric substrate $S_{n}$ is described by a Michaelis-Menten reaction occuring in a single step :

$$
S_{n} \longrightarrow n S_{1}
$$

where the maximal reaction velocity is supposed to be proportional to the bacterial density, $\bar{\alpha} \rho$.

This Michaelian kinetics is now generalized by considering a multi-step degradation 


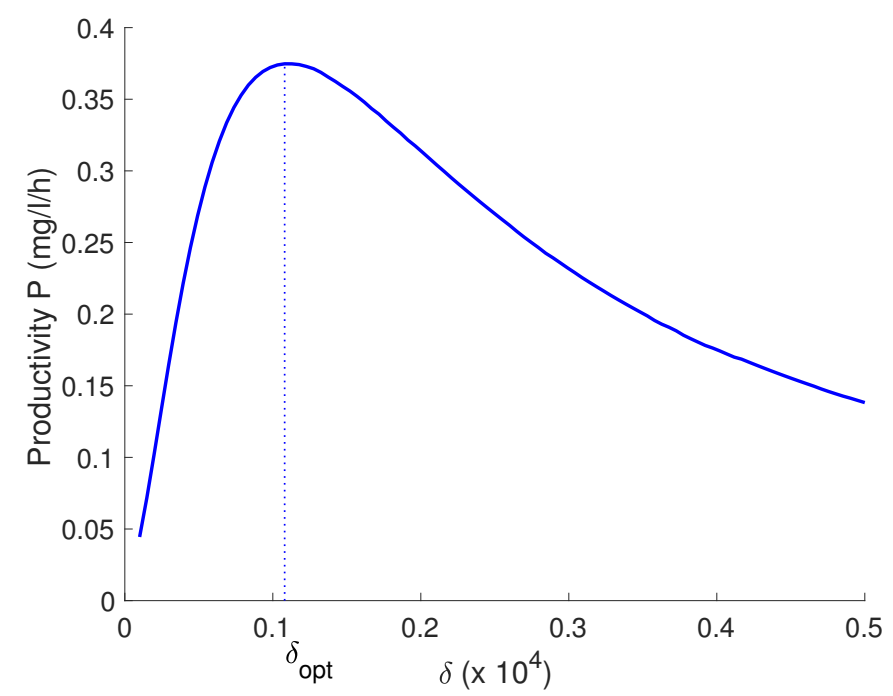

Figure 2: Productivities of the closed-loop controlled system (0.8), in function of control parameter $\delta$. The optimal parameter $\delta_{\text {opt }}$ is the abscissa corresponding to the maximal productivity. System parameters used in equations are the same as for Fig. 1.

of polymers, taking into account polymeric molecules with any intermediate lengths $l$ comprised between 2 and $n$.

Various models of biopolymer degradation are reported in the litterature ${ }^{19-24}$. One of the earliest mechanistic model of enzymatic depolymerisation ${ }^{19}$ treats the enzymatic hydrolysis of cellulose, where several enzymes attack the polymeric chain. Below a simplified version of this model has been re-derived, where only one enzyme is present. In order to simplify the kinetic equations, the mechanism of end-product inhibition is neglected, as it was found to be not essential in the modeling of decomposition of polygalacturonate one of us performed previously ${ }^{10}$.

Let us first describe the mechanism of polymer decomposition in a batch reactor $(D=$ 0 ), with no bacteria but containing a degradative enzyme whose total concentration is given by $E_{T}$. By supposing that the enzyme can bind and cut any sites of the polymer, 


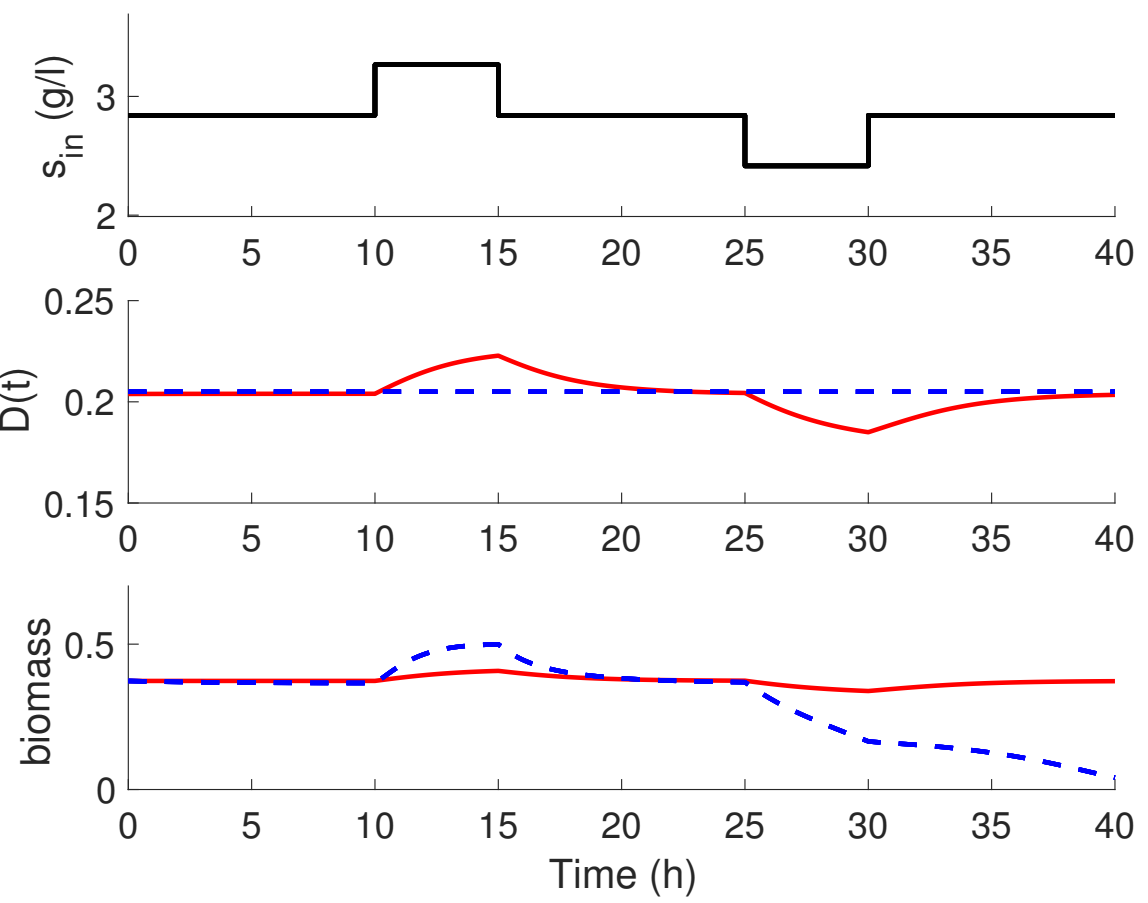

Figure 3: Comparison of the controlled (continuous red lines) and uncontrolled system (dashed blue lines) when it works at its optimal point in presence of a fluctuating input $s_{i n}$ of the chemostat. In absence of control ( $D=D_{c}$ is constant) a positive fluctuation of $s_{i n}$ is harmless, but a negative fluctuation can provoke washing out of the bioreactor. On the other hand, when the control law $D=\delta_{\text {opt }} \rho$ is applied (where $\delta_{o p t}$ corresponds to the optimal parameter giving the maximum of $P$ in Fig. 2), any fluctuation of the input $s_{i n}$ is compensated by a variation $D(t)$ allowing to recover the optimal functioning point when $s_{i n}$ retrieves its initial value. 
one considers a series of enzymatic reactions for the fragmentation of polymer $S_{l}$ of length $l(2 \leq l \leq n)$. For a polymer $S_{l}$ (constituted of $l$ monomers), $l-1$ complexes can be formed between the polymer and the enzyme. These considerations lead to the following chemical equations:

$$
S_{l}+E \underset{d_{m l}}{\stackrel{a_{m l} l}{=}} C_{m l} \stackrel{k_{m l}}{\longrightarrow} S_{l-m}+S_{m}+E,
$$

where $C_{m l}$ is the $E . S_{l}$ complex with the enzyme fixed on the $(m, m+1)$ dimer. Thus for a fixed $l \in\{2, \ldots, n\}$, the index $m$ varies from 1 to $l-1$. The kinetic parameters $a_{m l}, d_{m l}$ are, respectively, the association and the dissociation constants of enzymesubstrate complex, when the enzyme binds the $(m, m+1)$ dimer (starting from one either end) of a polymer of intermediate length $l$, and $k_{m l}$ is the maximal fragmentation rate of the same complex.

Denoting the molecular concentrations of species $E$ and $C_{m l}$ by $e$ and $c_{m l}$, the conservation law on the total enzyme gives:

$$
e_{T}=e+\sum_{l=2}^{n} \sum_{m=1}^{l-1} c_{m l} .
$$

The sum counts the free available enzyme $e$ and all enzyme-substrate complexes $c_{m l}$ which are supposed to sequestrate one enzyme molecule at a time. The dynamics of the molecular concentration $s_{l}$ of polymer $S_{l}$ is obtained by applying the law of mass action to the reaction scheme $(0.10)$ :

$$
\begin{aligned}
\frac{d s_{l}}{d t} & =\sum_{j=l+1}^{n}\left(k_{j l} c_{j l}+k_{j-l, j} c_{j-l, j}\right)-\sum_{m=1}^{l-1}\left(-a_{m l} s_{l} e+d_{m l} c_{m l}\right) \\
\frac{d c_{m l}}{d t} & =a_{m l} s_{l} e-d_{m l} c_{m l}-k_{m l} c_{m l}
\end{aligned}
$$

with $l=2, \ldots n$ and $m=1, \ldots, l-1$. Actually, eq.(0.12) with index $l=1$ describes the accumulation of monomers as the product of these cascade of enzymatic reactions. The usual quasi-steady assumption is considered here, stating that the dynamics of the 
complexes is much faster than the one of the substrates ${ }^{25}$. This approximation leads to replace the variables $c_{m l}$ by their stationary values, namely $c_{m l}=e s_{l} / K_{m l}$, where the $K_{m l}$ are the so-called Michaelis-Menten constants:

$$
K_{l m}=\frac{d_{l m}+k_{l m}}{a_{l m}}
$$

Moreover, one will assume that the kinetic rates $a_{m l}, d_{m l}$ and $k_{m l}$ describing chemical reactions (0.10) do not depend on $m$ and $l$, i.e. $a_{m l}=a, d_{m l=d}$ et $k_{m l}=k$. So, the Michaelis-Menten constants $K_{m l}$ for each chemical reactions described in equation (0.10) are identical and will be denoted $K_{M}$. This assumption of uniformity simplifies the conservation law (0.11) which can be written:

$$
e_{T}=e\left(1+\sum_{l=2}^{n}(l-1) \frac{s_{l}}{K_{M}}\right) .
$$

This simplification enables to express the steady state value of $c_{m l}$ as a function of the substrate variables $s_{l}$ :

$$
c_{m l}=e_{T} \frac{s_{l}}{K_{M}+\sum_{l=2}^{n}(l-1) s_{l}} .
$$

By substituting this expression of $c_{m l}$ into eq. (0.12), one obtains the system of equations governing the fragmentation dynamics of the substrates:

$$
\frac{d s_{l}}{d t}=k e_{T} \frac{\sum_{j=l+1}^{n} 2 s_{j}-(l-1) s_{l}}{K_{M}+\sum_{j=2}^{n}(j-1) s_{j}},
$$

where $l=n-1, \ldots, 1$. Let us observe that this latter expression generalizes the standard Michaelis-Menten kinetics with only one substrate (i.e. $n=l=2$ ) namely : $\dot{s_{2}}=-k e_{T} \frac{s_{2}}{K_{M}+s_{2}}$.

Now let us return to the description of these enzymatic reactions when they occur in a bioreactor containing degradative bacteria, and fed by a flow of polymeric substrate of length $n$, that is $D s_{i n}$. Furthermore, as previously the total enzyme concentration 
is supposed to be proportional to the bacterial density and the bacteria consume the monomer product $s_{1}$ for their growth. Then the state of the bioreactor evolves according to the following system of differential equations:

$$
\begin{aligned}
\frac{d s_{n}}{d t} & =D\left(s_{i n}-s_{n}\right)-\frac{k \rho}{K_{M}+\sum_{j=2}^{n}(j-1) s_{j}}(n-1) s_{n}, \\
\frac{d s_{l}}{d t} & =-D s_{l}+\frac{k \rho}{K_{M}+\sum_{j=2}^{n}(j-1) s_{j}}\left[\sum_{j=l+1}^{n} 2 s_{j}-(l-1) s_{l}\right], l=n-1, \ldots, 2, \\
\frac{d s_{1}}{d t} & =-D s_{1}+\frac{k \rho}{K_{M}+\sum_{j=2}^{n}(j-1) s_{j}}\left[\sum_{j=2}^{n} 2 s_{j}\right]-\gamma \mu\left(s_{1}\right) \rho \\
\frac{d \rho}{d t} & =\left(\mu\left(s_{1}\right)-D\right) \rho
\end{aligned}
$$

This system generalizes the minimal model (0.1). Nevertheless the next Section will show that the control method developed for the minimal model can be also applied to the larger system detailing the polymer fragmentation.

\section{Control in the fragmentation model}

Following the same development as in the proof of Theorem 1, and noting that in the detailed model the same relation (0.2) between $s_{1}^{*}$ and $D$ holds as in the minimal model, one can expect that the maximum steady-state productivity $D s_{1}$ is obtained for the highest dilution rate such that there exists a non-trivial equilibrium (this result is comforted by numerical simulations, see next Section). Operating the system at this point in open loop is risky, so the development of a control law is of particular interest. It will be shown that the control law proposed for the reduced system can be used for the whole model.

Theorem 4. Control law (0.7) globally stabilize System (0.16) towards the non-trivial 
equilibrium $E^{*}(\delta):=\left(s_{n}^{*}(\delta), \ldots, s_{1}^{*}(\delta), x^{*}(\delta)\right)$, where

$$
\begin{aligned}
& s_{n}^{*}(\delta)=\frac{\delta s_{i n}\left(K_{M}+R^{*}(\delta)\right)}{\delta\left(K_{M}+R^{*}(\delta)\right)+(n-1) k}, \\
& s_{l}^{*}(\delta)=\frac{2 k \sum_{j=i+1}^{n} s_{j}^{*}(\delta)}{\delta\left(K_{M}+R^{*}(\delta)\right)+(l-1) k}, l=n-1, \ldots, 2, \\
& s_{1}^{*}(\delta)=\frac{k \sum_{j=2}^{n} s_{j}^{*}}{\delta\left(K_{M}+R^{*}\right)}-\frac{\delta K_{1}+\gamma \bar{\mu}}{2 \delta}+\sqrt{\left[\frac{k \sum_{j=2}^{n} s_{j}^{*}}{\delta\left(K_{M}+R^{*}\right)}-\frac{\delta K_{1}+\gamma \bar{\mu}}{2 \delta}\right]^{2}+\frac{2 K_{1} k \sum_{j=2}^{n} s_{j}^{*}}{\delta\left(K_{M}+R^{*}\right)}} \\
& x^{*}(\delta)=\frac{\bar{\mu} s_{1}^{*}}{\delta\left(K_{1}+s_{1}^{*}\right)} . \\
& \text { with } R^{*}(\delta)=\frac{\delta(n-1) s_{i n}-k-\delta K_{M}+\sqrt{\left(\delta(n-1) s_{i n}-k-\delta K_{M}\right)^{2}+4 K_{M} \delta^{2}(n-1) s_{i n}}}{2 \delta}
\end{aligned}
$$

Proof. System (0.16) under Control law (0.7) becomes:

$$
\begin{aligned}
\frac{d s_{n}}{d t} & =\rho\left[\delta\left(s_{i n}-s_{n}\right)+\frac{k}{K_{M}+\sum_{j=2}^{n}(j-1) s_{j}}\left(-(n-1) s_{n}\right)\right] \\
\frac{d s_{l}}{d t} & =\rho\left[-\delta s_{l}+\frac{k}{K_{M}+\sum_{j=2}^{n}(j-1) s_{j}}\left(\sum_{j=i+1}^{n} 2 s_{j}-(l-1) s_{l}\right)\right], l=n-1, \ldots, 2 \\
\frac{d s_{1}}{d t} & =\rho\left[-\delta s_{1}+\frac{k}{K_{M}+\sum_{j=2}^{n}(j-1) s_{j}}\left(\sum_{j=2}^{n} 2 s_{j}\right)-\gamma \mu\left(s_{1}\right)\right] \\
\frac{d \rho}{d t} & =\rho\left[\mu\left(s_{1}\right)-\delta \rho\right]
\end{aligned}
$$

Similarly to the proof of Theorem 2, $\rho(t)$ can be proved to be lower bounded, and then System (0.18) can be rewritten under a cascade structure to prove the convergence to the equilibrium point.

Let us define $R=\sum_{l=1}^{n}(l-1) s_{l}$, whose dynamics under Control law (0.7) is given by:

$$
\frac{d R}{d t}=\rho\left[\delta(n-1) s_{i n}-\delta R-\frac{k}{K_{M}+R} R\right] .
$$

It follows that $R(t)$ is lower bounded by $\underline{R}:=\min \left(R(0), R^{*}(\delta)\right)$. 
Now given that $\mu\left(s_{1}\right) \leq \bar{\mu} s_{1} / K_{1}$ and

$$
\sum_{j=2}^{n} s_{j} \geq \sum_{j=2}^{n} \frac{j-1}{n-1} s_{j}=\frac{R}{n-1}
$$

we get

$$
\frac{d s_{1}}{d t} \geq \rho\left[\frac{2 k R}{(n-1)\left(K_{M}+R\right)}-s_{1} \delta-\gamma \mu\left(s_{1}\right)\right] \geq \rho\left[\frac{2 k \underline{R}}{(n-1)\left(K_{M}+\underline{R}\right)}-s_{1}\left(\delta+\gamma \bar{\mu} / K_{1}\right)\right] .
$$

Thus, $s_{1}(t)$ admits a lower bound denoted $\underline{s_{1}}$. This finally gives:

$$
\frac{d \rho}{d t} \geq \rho\left[\mu\left(\underline{s_{1}}\right)-\delta \rho\right]
$$

which allows us to conclude that $\rho(t)$ is lower bounded. One can introduce again the time change $\tau=\int_{0}^{t} \rho(x) d x$. Considering additionally the variable $R$, System (0.18) becomes:

$$
\begin{aligned}
\frac{d R}{d \tau} & =\delta(n-1) s_{i n}-\delta R-\frac{k}{K_{M}+R} R \\
\frac{d s_{n}}{d \tau} & =\delta\left(s_{i n}-s_{n}\right)+\frac{k}{K_{M}+R}\left(-(n-1) s_{n}\right) \\
\frac{d s_{l}}{d \tau} & =-\delta s_{l}+\frac{k}{K_{M}+R}\left(\sum_{j=l+1}^{n} 2 s_{j}-(l-1) s_{l}\right), l=n-1, \ldots, 2, \\
\frac{d s_{1}}{d \tau} & =-\delta s_{1}+\frac{k}{K_{M}+R}\left(\sum_{j=2}^{n} 2 s_{j}\right)-\gamma \mu\left(s_{1}\right) \\
\frac{d \rho}{d \tau} & =\mu\left(s_{1}\right)-\delta \rho .
\end{aligned}
$$

Given the cascade structure and considering the unique positive solution of system (0.18), that is $E^{*}(\delta)=\left(s_{n}^{*}(\delta), \ldots, s_{1}^{*}(\delta), x^{*}(\delta)\right)$, the equilibrium $E^{*}(\delta)$ is deduced to be globally stable. 


\section{Numerical simulations and discussion}

In this section, we present numerical simulations of the system and report some properties of the polymer distribution. Let us consider the system of enzymatic decomposition of polymers in a bioreactor, described by eqs.(0.16). Parameter values are chosen identical to the ones used to compute the steady states of the minimal model, as represented earlier in Fig. 1.

Let us note that when comparing simulations performed with different values of maximal polymer length $n$, the bioreactor is assumed to be fed only by polymers of length $n$, with a fixed mass concentration. This means that if $\sigma_{i n}$ denotes such mass concentration, the corresponding molar concentration depends on $n$ as $s_{i n}=\frac{\sigma_{i n}}{n \mathcal{P}}$, where $\mathcal{P}$ is the molecular weight of the monomer. On the other hand, the initial state of the bioreactor will be chosen with no polymer $\left(s_{l}=0, l=1, \ldots, n\right)$ but sufficient biomass to avoid the washout of the bioreactor. Let us take the convention to choose the initial value of $\rho$ equivalent to the feeding concentration : $\rho(0)=n s_{i n} / \gamma$.

Then, starting from 0 , the dilution coefficient $D$ is increased over a given range. For each $D$, one numerically computes the time evolution of the bioreactor until it reaches its steady state. Figure 4 shows the stable branches of non trivial steady states of the biomass, for three different values of polymer length $n \in\{2,10,100\}$. As studied above for the minimal model, each branch of steady states ends on a limit point corresponding to a saddle-node bifurcation for a critical value $D_{c}$ depending on $n$. For $D>D_{c}$ only the trivial solution $\rho=0$ exists. In Fig. 4 , the rightmost branch corresponds to $n=2$. It is identical to the one drawn on the third panel of Fig. 1. A similar behavior is obtained for the other branches, computed respectively with $n=10$ and 100. Moreover the branches tend to accumulate on the same line when $n$ is increased. For example one checked that the steady states computed with $(n=200)$ would super- 
pose on the same curve as $n=100$. Therefore the critical value $D_{c}(n)$ of the dilution coefficient converges to an infimum $\bar{D}_{c}$ when $n$ increases.

As already noted, the knowledge of $D_{c}$ is useful because it determines the productivity and so the optimal efficiency of the bioreactor by eq. (0.6)). The existence of a lower limit for $D_{c}$, gives a lower limit of the maximal efficiency, which holds for any size of polymer length $n$ :

$$
\bar{\eta}_{\max }=\frac{\bar{D}_{c} K_{1}}{n s_{i n}\left(\bar{\mu}-\bar{D}_{c}\right)}
$$

In the general system $(0.16), D_{c}$ should be computed numerically. On the other hand, the existence of a lower limit for $D_{c}$ can be analytically worked out with the approximate value $\mathcal{D}_{c}$ obtained in eq. (0.5), in the case where the polymer decomposition occurs in a single step and where $D \ll \mu$. Indeed, considering $s_{i n}=\frac{\sigma_{i n}}{n \mathcal{P}}$ in this equation, one can compute the limit of $\mathcal{D}_{c}(n)$ when $n \rightarrow \infty$. This provides an approximate value of the optimal efficiency as follows :

$$
\bar{\eta}_{\max } \approx \frac{\chi}{(\sqrt{\chi}+1)^{2}} \quad \text { with } \quad \chi=\frac{\bar{\alpha} K_{1}}{\gamma \bar{\mu} K_{M}}
$$

So, in this approximation one obtains a simple analytical result which can be interpreted as follows. One sees that the optimal efficiency of the enzymatic decomposition of polymers varies between 0 and 1 , increasing with the dimensionless parameter $\chi$ defined by eq. (0.20). This lumped parameter reflects the balance between two competing processes occurring simultaneously in the system. The first process is the outflow of the monomer out of the bioreactor, which depends on the amount of hydrolysed polymer by the bacterial enzyme. This amount relies on the activity of the degradating enzyme, and can be quantified by the kinetic factor $\left(\bar{\alpha} / K_{M}\right)$. The second process is the metabolization of the monomeric substrate by the bacteria, which is analog to another enzymatic reaction. So it can be quantified by the factor $\left(\gamma \bar{\mu} / K_{1}\right)$. Therefore $\chi$ 
can be interpreted as the ratio between these two kinetic factors, and it is large when the bacterial enzymatic activity is larger than the bacterial metabolization. Another point of view is to say that this optimal efficiency is closer to 1 when each bacteria produces a lot of enzyme $(\bar{\alpha} / \gamma \bar{\mu}$ is large), and when each enzyme molecule has a higher affinity than the bacteria for the polymer substrate $\left(K_{1} / K_{M}\right.$ large $)$. A last feature, mentioned in the framework of the approximation of $D_{c}$ by (0.5), is the computation of the limit value of the normalized biomass. Using eq. (0.20), one can calculate :

$$
\frac{\rho^{*}\left(D_{c}\right)}{\rho_{\max }^{*}}=\frac{\sqrt{\chi}}{(\sqrt{\chi}+1)^{2}}
$$

Thus, the critical biomass decreases asymptotically like $\chi^{-\frac{1}{2}}$. In conclusion, eqs. (0.20)(0.21) show that when $\chi$ becomes large the optimal functioning of the bioreactor tends to the highest efficiency. But at the same time the stationary bacterial density becomes quite low, and therefore a severe risk of washout appears in the uncontrolled system. Hence the need, in this situation, to implement the feedback loop described above.

Next, let us explore the steady state distribution of polymer length as $D$ increases. This feature of the system can be investigated numerically. To fix the idea, we consider an example with the maximal polymer length $n=10$. Then, Fig.5 illustrates the profile of polymer lengths when $D=D_{c}$ and for 2 other lower values of $D$. It can be seen that in the 3 cases, the proportion of monomers is always maximal, and furthermore it grows by increasing $D$. This property motivates further the fact that the optimal point of functioning of this system is reached for $D=D_{c}$, and therefore the necessity to implement a control method since the optimal steady state is structurally unstable without control. Thus, we consider the chemostat system regulated with the simple control law (0.7), namely $D(t)=\delta \rho(t)$, so that the equations of the system become (0.18). In order to determine the optimal $\delta_{\text {opt }}$, the curves of steady states of $\rho$ in function of $D$ 

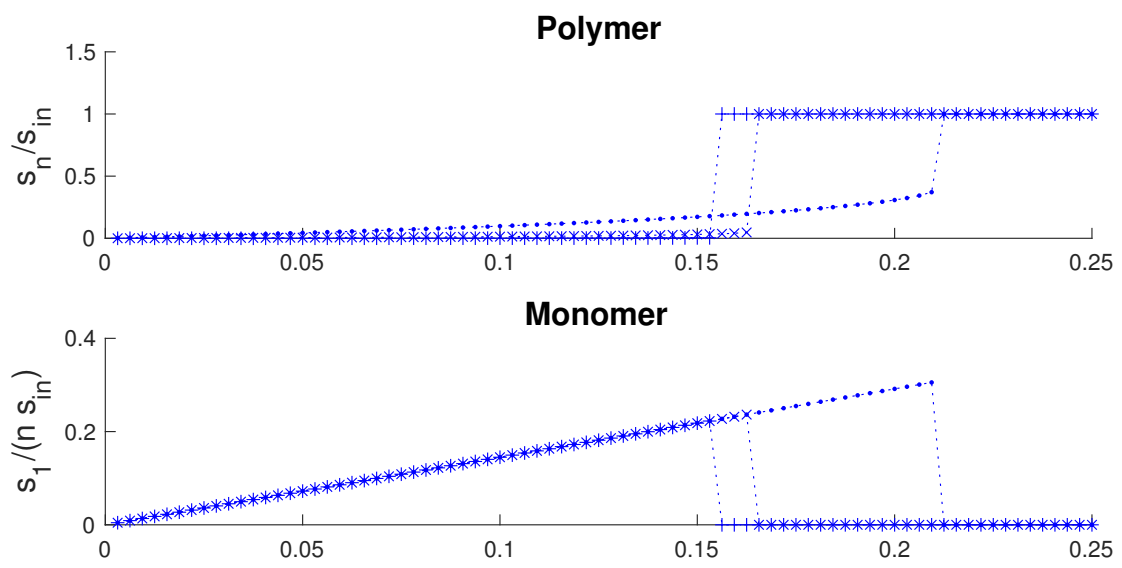

Biomass

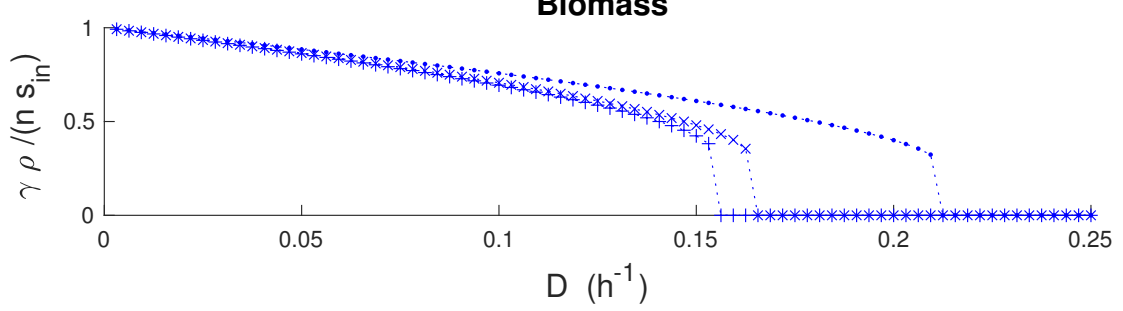

Figure 4: Bifurcation diagrams of the non-trivial stable steady states of the concentrations of $n$-polymers, of monomers and biomass in the chemostat, for different values of $n$. The symbols represent respectively $(\bullet) n=2,(\mathrm{x}) n=10,(+) n=100$. Parameter values as in Fig. 1.

are first computed, as done in Fig. 4, for example for $n=10$. Then $\delta_{o p t}=D_{c} / \rho\left(D_{c}\right)$ is computed, which provides the control parameter that makes the steady states to be stable. Let us note that one advantage of this control method is that the simulation can start with all the polymer variables equal to 0 and an arbitrary (but non zero) value of the initial biomass $\rho(0)$. For example, $\rho(0) / \rho_{\max }=0.1$ is considered, a value which would rapidly lead to washout of the uncontrolled chemostat. Then, the time evolution of the variables is represented in Fig. 6, until they reach their optimal steady states, whose profile (in blue) is indeed equivalent to the maximal profile displayed in Fig. 5. 


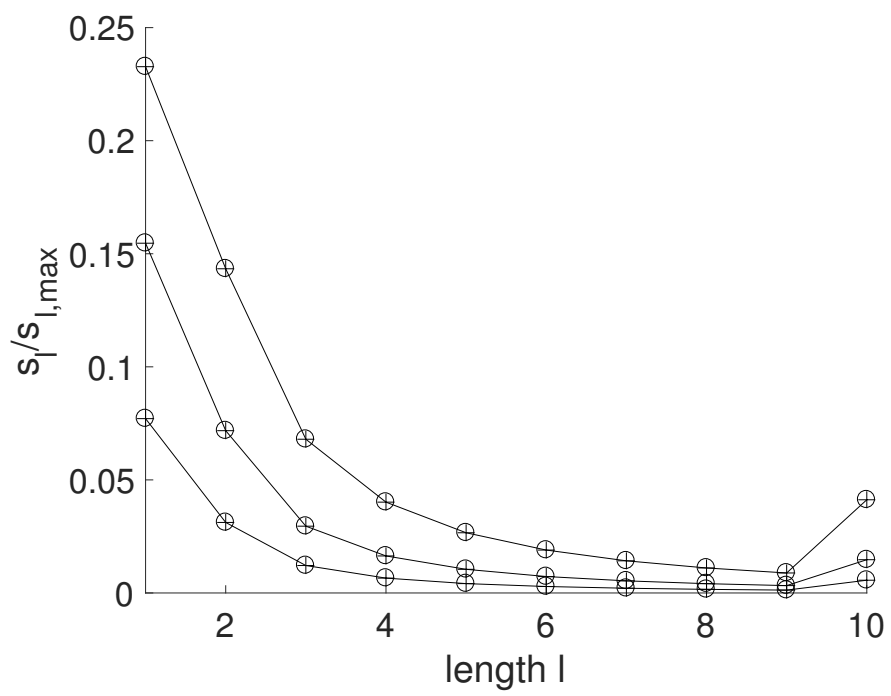

Figure 5: Steady state distribution of polymers for 3 values of $D \in$ $\left\{D_{c} / 3,2 D_{c} / 3, D_{c}\right\}$. The profile increases with $D$, with the maximal values reached for $D_{c}$. The concentrations of monomers are maximal in all cases. Parameter values are as in Fig. 1.

Finally, let us briefly discuss the robustness of the control law. As a matter of fact, theorem 4 guarantees that the optimal control law, $D(t)=\delta_{\text {opt }} \rho(t)$, is robust with respect to state fluctuations, since with this feedback law there is a unique steady state which is globally stable. Nevertheless, it is interesting to probe the sensitivity of the obtained optimal productivity $P_{o p t}$, and the corresponding control coefficient $\delta_{o p t}$, with respect to system's parameters. Numerical results reported on Table 1 show that most of the sensitivities are lower than 1 , except for sensitivities of the optimal productivity with respect to parameters $\alpha$ and $\gamma$, which are the same in absolute value, i.e. 1.14. Therefore, we can conclude that the proposed control law is not critically sensitive to parameter values. The numerical computation of sensitivities is reported on Table 1 for polymer length $n=10$, but one checked that these values are almost the same for 


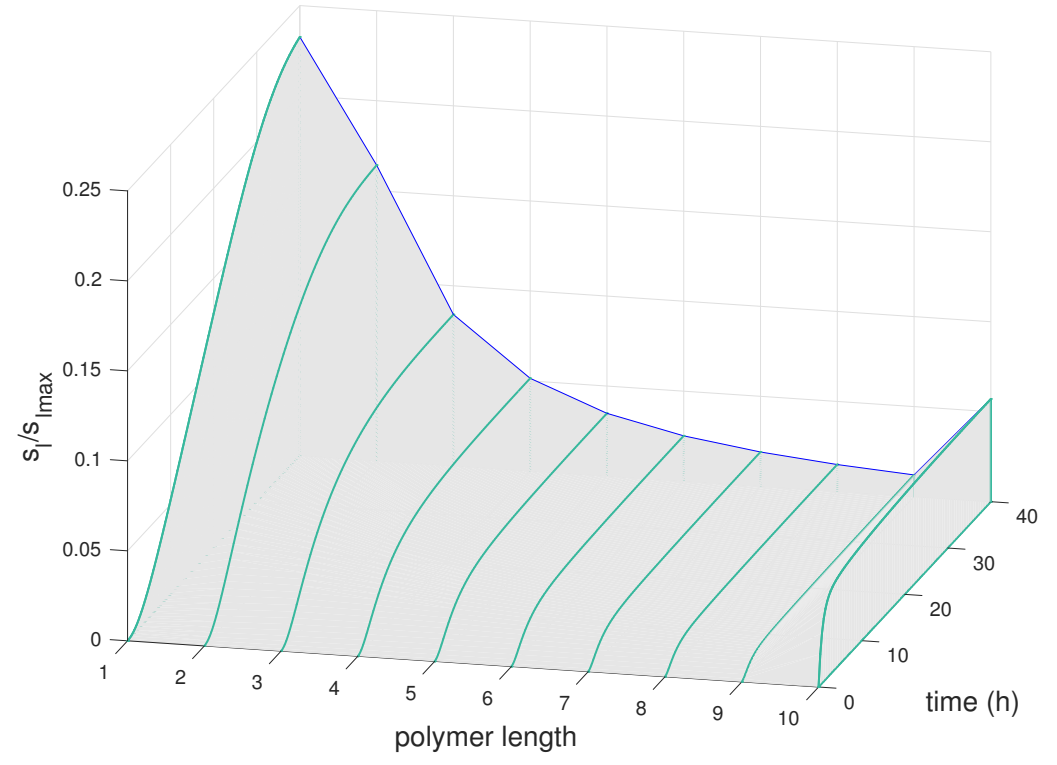

Figure 6: Time-evolution of the distribution of polymer concentrations including all the intermediate size between the maximal polymer length $n=10$ and monomers. The system is regulated by the law $D=\delta_{\text {opt }} \rho$, such that its dynamics is described by eqs. (0.18). Parameters are as those in Fig. 1, and the initial condition is $s_{l}=0, l=$ $1, \ldots, n$, and $\rho(0) / \rho_{\max }=0.1$

larger $n$.

\section{Conclusion}

The content of this paper studies a simple model and its extension, describing the enzymatic decomposition of a polymeric substrate in a bioreactor. In an open loop configuration, the polymer feeds, with a constant dilution rate $D$, a chemostat hosting bacteria able to synthesize the degradative enzyme. The decomposed polymer is partly consumed by the bacteria in order to maintain the biomass at a constant level in the 


\begin{tabular}{|c|c|c|}
\hline \multirow{2}{*}{$\begin{array}{l}\text { Parameters } \\
p \\
\end{array}$} & \multicolumn{2}{|c|}{ Sensitivities } \\
\hline & $\frac{\partial \log P_{o p t}}{\partial \log p}$ & $\frac{\partial \log \delta_{o p t}}{\partial \log p}$ \\
\hline$k$ & 1.14 & 0.60 \\
\hline$\mu$ & -0.14 & 0.30 \\
\hline$\gamma$ & -1.14 & -0.30 \\
\hline$K_{M}$ & -0.91 & -0.60 \\
\hline$K_{1}$ & 0.14 & -0.30 \\
\hline
\end{tabular}

Table 1: The robustness of the control law is tested by numerically computing the sensitivities of the optimal productivity $P_{o p t}$ and of the control coefficient $\delta_{o p t}$, locally around a set of reference parameter values (same as those in Fig. 5 and 6). The numerical method is as follows : the value of each system's parameter $p$ is increased by $0.5 \%$; then, the corresponding relative increases (or decreases) of $P_{o p t}$ and $\delta_{o p t}$ are computed; finally these relative variations are divided by $0.5 \%$.

chemostat, while the non-consumed part of monomers are extracted from the reactor as the product of interest. The analysis of the model shows that the productivity of this process augments when $D$ is increased. On the other hand, at the same time the biomass decreases, and reaches a positive value for a limit point $D=D_{c}$. For $D>D_{c}$, the chemostat supports no other steady state than the trivial zero-biomass state. Therefore, in the open loop configuration, the optimal productivity point of the system appears to be a bifurcation point which is intrinsically not robust with respect to fluctuations of the state or of the parameters. Thus, the main interest of our study is to predict that by measuring continuously the optical density of the chemostat and by considering a proportional feedback on the dilution rate, the structurally unstable 
point $D=D_{c}$ can be turned in a globally stable steady point. This was proved first in a minimal 3-variables model, and then, generalized in a detailed model of enzymatic decomposition of polymers.

This control law on $D$ can be especially useful in situations where the enzyme synthesized by the bacteria are very efficient in hydrolyzing the polymeric substrate. Indeed, in this case one expects that only a small bacterial colony is sufficient to maximally produce the monomer, and therefore, the limit steady state value of the bacterial density can become close to 0 . This feature, which is clear intuitively, was discussed analytically in eqs. (0.20)-(0.21), using the minimal model with simplified hypothesis. Thus, in the uncontrolled system, the bioreactor is close to the washout point toward which it will eventually go in presence of perturbations. With the help of the designed feedback loop, this washout is avoided and moreover the optimal productivity is maintained.

An extension of this work will be to consider the addition of a second substrate which can be used directly by the biomass growth in order to prevent the bacteria to consume the decomposed polymer of interest. Another refinement of the model is to relax the simplified hypothesis that the produced enzyme is proportional to the bacterial density. A more realistic description of the system would be to incorporate in the model the regulatory mechanisms of the gene coding for the degradative enzyme. For instance, this step can be developed in the case of the pectin decomposition by Dickeya dadantii whose genetic regulation has been modelised in ${ }^{10}$.

Even though the method we propose is able to stabilize the optimal point, the implementation of our process would need to compute a priori the optimal proportionality coefficient. Furthermore, biological parameters of the system can fluctuate in time. Therefore another perspective of this work is to implement an adaptive control in order to automatically track the point of optimal productivity. 


\section{Acknowledgements}

This work was initiated in the framework of the project PEPS BMI Pectolyse funded in 2014. JAS acknowledges a sabbatical stay in the team Biocore of Inria, from January to June 2017. W. Nasser and S. Reverchon are acknowledged for stimulus discussions about the example system of pectin degradation by $D$. dadantii, and for providing us with bibliographical references.

\section{References}

[1] D. Klemm, B. Heublein, H.-P. Fink, and A. Bohn, "Cellulose: Fascinating biopolymer and sustainable raw material," Angewandte Chemie International Edition, vol. 44, no. 22, pp. 3358-3393, 2005.

[2] B. D. Solomon, J. R. Barnes, and K. E. Halvorsen, "Grain and cellulosic ethanol: History, economics, and energy policy," Biomass and Bioenergy, vol. 31, no. 6, pp. $416-425,2007$.

[3] R. Kumar, S. Singh, and V. Singh, Om, "Bioconversion of lignocellulosic biomass: biochemical and molecular perspectives," J Ind Microbiol Biotechnol, vol. 35, pp. 377-391, 2008.

[4] L. Canilha, A. K. Chandel, T. Suzane dos Santos Milessi, F. A. F. Antunes, W. Luiz da Costa Freitas, M. das Graças Almeida Felipe, and S. S. da Silva, "Bioconversion of sugarcane biomass into ethanol: An overview about composition, pretreatment methods, detoxification of hydrolysates, enzymatic saccharification, and ethanol fermentation," Journal of Biomedicine and Biotechnology, vol. 2012, 2012. 
[5] M. Edwards and J. Doran-Peterson, "Pectin-rich biomass as feedstock for fuel ethanol production," Applied Microbiology and Biotechnology, vol. 95, no. 3, pp. 565-575, 2012.

[6] M. Sainz, "Commercial cellulosic ethanol: The role of plant-expressed enzymes," in Biofuels (D. Tomes, P. Lakshmanan, and D. Songstad, eds.), pp. 237-264, Springer New York, 2011.

[7] B. Yang and C. E. Wyman, "Pretreatment: the key to unlocking low-cost cellulosic ethanol," Biofuels, Bioprod. Bioref., vol. 2, 2008.

[8] T. Li, J. Zeng, D. Singh, and S. Chen, "New insights into microbes in the midgut of termite coptotermes formosanus," J Bioremed Biodeg, vol. 5, 2014.

[9] N. Hugouvieux-Cotte-Pattat, G. Condemine, W. Nasser, and S. Reverchon, "Regulation of pectinolysis in erwinia chrysanthemi," Annu. Rev. Microbiol., vol. 50, 1996.

[10] W. Kepseu, J.-A. Sepulchre, S. Reverchon, and W. Nasser, “Toward a quantitative modeling of the synthesis of the pectate lyases, essential virulence factors in dickeya dadantii," J. of Biol. Chem., vol. 285, 2010.

[11] J. Monod, Recherches sur la croissance des cultures bacteriennes. Herman and Cie, Paris, 1942.

[12] B. Tang and G. Wolkowicz, "Mathematical models of microbial growth and competition in the chemostat regulated by cell-bound extracellular enzymes," J. Math. Biol., vol. 31, pp. 1 -23, 1992.

[13] M. A. Henson and D. E. Seborg, Nonlinear process control. Prentice Hall PTR Upper Saddle River, New Jersey, 1997. 
[14] K. M. Hangos, J. Bokor, and G. Szederkényi, Analysis and control of nonlinear process systems. Springer Science \& Business Media, 2006.

[15] L. Mailleret, O. Bernard, and J.-P. Steyer, "Nonlinear adaptive control for bioreactors with unknown kinetics," Automatica, vol. 40, no. 8, pp. 1379-1385, 2004.

[16] L. Mailleret, J.-L. Gouzé, and O. Bernard, "Nonlinear control for algae growth models in the chemostat," Bioprocess and biosystems engineering, vol. 27, no. 5, pp. 319-327, 2005.

[17] E. D. Sontag, Mathematical control theory: deterministic finite dimensional systems, vol. 6. Springer Science \& Business Media, 2013.

[18] M. Vidyasagar, Nonlinear Systems Analysis. Classics in Applied Mathematics, Society for Industrial and Applied Mathematics, 2002.

[19] M. Okazaki and M. Moo-Young, "Kinetics of enzymatic hydrolysis of cellulose: analytical description of a mechanistic model," Biotechnology and bioengineering, vol. 20, no. 5, pp. 637-663, 1978.

[20] A. Tayal and S. A. Khan, "Degradation of a water-soluble polymer: molecular weight changes and chain scission characteristics," Macromolecules, vol. 33, no. 26 , pp. 9488-9493, 2000.

[21] Y. Cheng, K. M. Brown, and R. K. Prud'homme, "Preparation and characterization of molecular weight fractions of guar galactomannans using acid and enzymatic hydrolysis," International journal of biological macromolecules, vol. 31, no. 1, pp. 29-35, 2002.

[22] S. Mahammad, R. K. Prud'homme, G. W. Roberts, S. A. Khan, et al., "Kinetics of 
enzymatic depolymerization of guar galactomannan," Biomacromolecules, vol. 7, no. 9, pp. 2583-2590, 2006.

[23] P. Bansal, M. Hall, M. J. Realff, J. H. Lee, and A. S. Bommarius, "Modeling cellulase kinetics on lignocellulosic substrates," Biotechnology advances, vol. 27, no. 6, pp. 833-848, 2009.

[24] S. E. Levine, J. M. Fox, H. W. Blanch, and D. S. Clark, "A mechanistic model of the enzymatic hydrolysis of cellulose," Biotechnology and bioengineering, vol. 107, no. 1, pp. 37-51, 2010.

[25] A. Cornish-Bowden, "Chapter 2 - introduction to enzyme kinetics," in Fundamentals of Enzyme Kinetics (A. Cornish-Bowden, ed.), pp. 16 - 38, ButterworthHeinemann, 1979. 